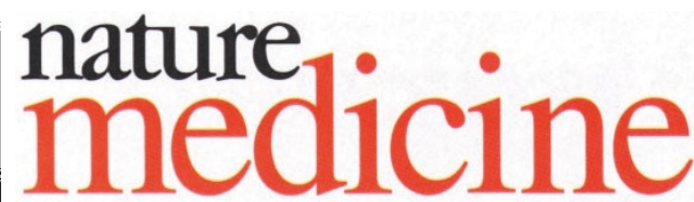

VOLUME 4 • NUMBER 6 • JUNE 1998

\title{
Molecular medicine growing pains
}

Despite the exciting subject and professional organization, last month's Congress of Molecular Medicine (Berlin, May 7th-9th) did little to promote the concept of an international community of biomedical researchers. The meeting is still young and there is as yet no well established meeting to compete with it. The organizers must quickly consider the aims of their meeting and how best to accomplish them.

Traditionally, the disciplines of science and medicine have not been close. In most academic settings, separate faculties of science and medicine still exist, often duplicating areas of interest. Whereas the faculties of science have promoted research in pursuit of knowledge, some of which can be applied to medical problems, the medical faculties have focused quite specifically on the patient, drawing on science when needed. The two groups have developed their own professional societies, conferences and journals and there is little crossover within these venues, each clearly dominated by either M.D.'s or Ph.D.'s

Now, and despite the different traditions and infrastructures, this redundant distinction is being discarded. The emerging discipline of molecular medicine (or biomedical research, depending on your persuasion) is drawing the science and medical communities so close as to make them virtually indistinguishable in many areas-are molecular studies of the breast cancer-predisposing BRCA1 genes scientific or medical endeavors? Likewise, how do we classify the exciting (although recently overhyped) anti-angiogenesis work that offers hope for the treatment and prevention of tumors? In fact, it really does not matter whether this genre of investigation is classified one way or the other. What matters is that this long- term strategy towards better health is recognized and further developed.

That this new discipline of molecular medicine is gaining acceptance is evidenced by the emergence of new societies and journals. This journal was launched three and a half years ago to address the needs of a growing community of molecular medicine. The UK recently announced the establishment of the Academy of Medical Sciences (see Nature Medicine 4, 375) and Italy has established its own society of molecular medicine. In the US, the journal Molecular Medicine was launched as a flagship of a new Molecular Medicine Society (based at the Picower Institute) and the European-based Joumal of Molecular Medicine was launched three years ago. It is gratifying to note, that by and large, these new ventures cannot be slotted easily into either the traditional scientific or medical communities. Instead, they reflect a partnership. Nature Medicine, for example, has a 50/50 split between M.D.- and Ph.D.-qualified readers. Just a few years ago such a statistic would have been unheard of. This hybridization of science and medicine is at the heart of molecular medicine and those of us who believe that the most exciting and important aspect of the life sciences is in its application to health see it as a very natural union.

Despite the new journals and societies, still lacking is a major international conference reflecting the interests of this emerging community. Good conferences are much more than just a venue to exchange new findings. They provide a focal point for a community-a time to take stock; to establish new collaborations; to challenge old ideas and consider new ones; to agree future directions and broaden one's perspective from the often narrow confines of the individual's day-to-day work. Last year, the first Congress of Molecular Medicine seemed ready to fulfill this role for the biomedical research community. Attracting nearly 2,000 participants, it was considered an excellent start and full of promise for the years to come. The second congress, however, suffered a considerable drop in attendance and a common perception that the meeting was not an international event.

The organizing committee should be clear on the aims of the meeting. If it was intended to showcase German biomedical research, the organizers are to be congratulated for putting together an impressive and wide-ranging meeting. If, on the other hand, it is intended that this meeting develops into the main international biomedical research meeting, they should think twice before holding it in the same city (Berlin) each year and making it the venue for many national organizations. (Much of last month's meeting was devoted to the 23 symposia organized by German societies.) Harald Stein, the organizer of the 1999 meeting, tells us that the Berlin Molecular Medicine Association has "decided to host the Congress on a yearly basis". Laudable as this commitment to the field is, it is not the action of "a truly international undertaking" as Stein intends. Indeed it will be very difficult to achieve an international status given this organizational framework.

In the closing plenary address, the 1994 Nobel laureate Martin Rodbell, in an analogy to cell signaling, stressed the importance of communication and social intercourse. His impression was that many scientific communities had lost this important skill and he hoped that the congress would help re-establish it. It is now incumbent upon Stein and his colleagues to decide just what level of communication the Congress of Molecular Medicine aspires to and how best to achieve it. 